\title{
CHARACTERISTIC OF A DIGITAL CORRELATION RADIOMETER BACK END WITH FINITE WORDLENGTH
}

\author{
Sayak K. Biswas ${ }^{1}$, David W. Hyde ${ }^{2}$, Mark W. James ${ }^{2}$ and Daniel J. Cecil ${ }^{2}$ \\ ${ }^{1}$ Universities Space Research Association, Huntsville, Al 35805 \\ ${ }^{2}$ NASA Marshall Space Flight Center, Huntsville, Al 35805
}

\begin{abstract}
The performance characteristic of a digital correlation radiometer signal processing back end (DBE) is analyzed using a simulator. The particular design studied here corresponds to the airborne Hurricane Imaging radiometer which was jointly developed by the NASA Marshall Space Flight Center, University of Michigan, University of Central Florida and NOAA. Laboratory and flight test data is found to be in accord with the simulation results. Overall design seems to be optimum for the typical input signal dynamic range. It was found that the performance of the digital kurtosis could be improved by lowering the DBE input power level. An unusual scaling between digital correlation channels observed in the instrument data is confirmed to be a DBE characteristic.
\end{abstract}

Index Terms - digital radiometer, HIRAD, digital correlation, kurtosis, correlator.

\section{INTRODUCTION}

In modern microwave radiometer systems used for interferometric, polarimetric and/or spectrometric measurements of natural thermal radiation, digital signal processing offers many practical advantages [1]. The interferometric measurement requires signals from many different antennas to be correlated whereas signal from the same antenna at two different polarizations is correlated for the polarimetric measurements. For spectrometric measurements one option is to correlate the antenna signal with a time-delayed version of itself to map out the autocorrelation function. Then by Fourier transformation the power spectrum can be obtained. The other method is to divide the radiometer passband into multiple subbands using polyphaser filter bank for spectral measurements. Polyphase filter banks can also be used for radio-frequency interference (RFI) mitigation [2]. In many modern radiometric systems combinations of these techniques are implemented. Furthermore digital correlation calls for analytical signals (Inphase, I \& Quadrature, Q signals) to be derived from the received antenna signal. This can also be implemented

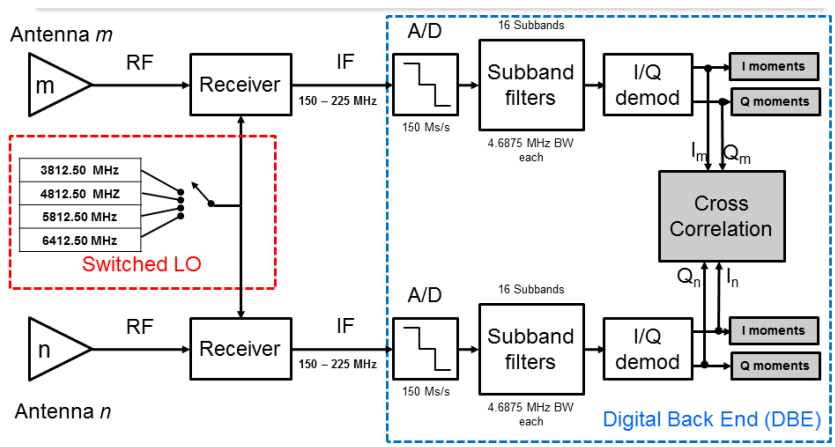

Figure 1: HIRAD system block diagram with DBE

digitally using Hilbert Transform (HT). Due to the advances in the development of modern high speed FPGAs (Field Programmable Gate Array) different signal processing schemes can be implemented and investigated using the same hardware. This offers to a great amount of flexibility.

Digitization involves sampling of the voltage signal at periodic interval and quantizing the sampled values so that each can be represented by a finite number of bits. The quantization effect on digital correlation has been studied extensively [3-13] but these studies does not include end-toend effect of finite bit truncation along the Digital Signal Processing (DSP) chain, if further processing of the quantized signal involved before computation of the digital correlation. For analytical signals, there may be a gain imbalance between I and Q channels because of HT filtering and bit truncation [14] which might also affect the correlation between quadrature channels. Here we present a study of a radiometer digital back end (DBE) which digitizes a band limited Intermediate Frequency (IF) Gaussian noise signal, performs polyphase filtering, I/Q demodulation and finally computes cross-correlation and statistical moments. The particular design studied here corresponds to the DBE of the airborne Hurricane Imaging Radiometer (HIRAD) jointly developed by NASA Marshall Space Flight Center, University of Michigan, University of Central Florida and NOAA [15].

\section{HIRAD DBE}




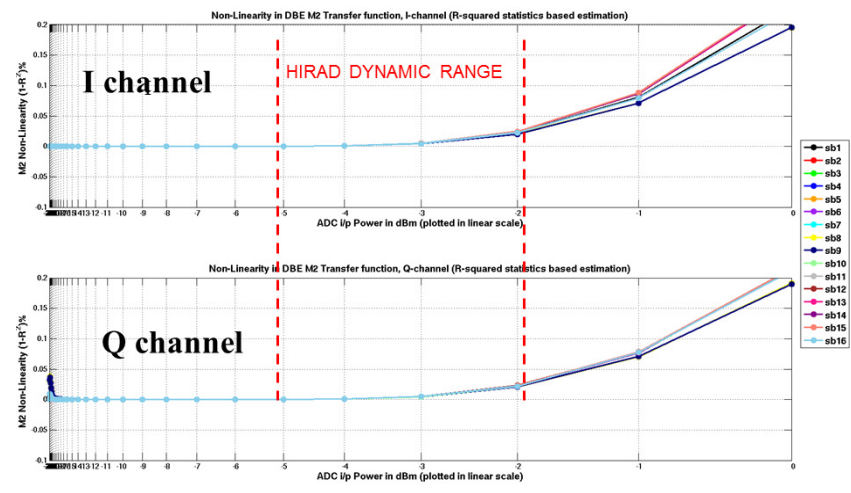

Figure 2: DBE non linearity (\%) vs. input power (dBm)

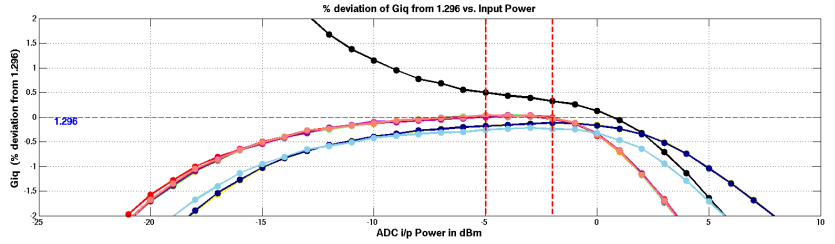

Figure 3: Giq deviation (\%) from 1.296 vs. input power

HIRAD instrument uses interferometric aperture synthesis technique to create high resolution cross-track brightness temperature image at 4.0, 5.0, $6.0 \& 6.6 \mathrm{GHz}$ using multiple fan beam antenna arranged in a thinned array configuration [16]. Each antenna is connected to a dedicated receiver which sequentially translates four RF passbands to a common $75 \mathrm{MHz}$ band centered at $187.5 \mathrm{MHz}$ by switching local oscillator (LO) frequency. This Intermediate Frequency (IF) signal is digitized by the DBE for further processing. The entire DBE is implemented in FPGA and its configuration is identical for all four RF frequencies. A schematic of a pair of antenna channels in HIRAD system is shown in Fig. 1 with the DBE inside the dashed box. The Analog to Digital Converter (ADC) samples the 150-225 $\mathrm{MHz}$ IF signal at Nyquist Rate $(150 \mathrm{MSa} / \mathrm{s})$ with 12 bit resolution. However, lower 5 bits are discarded and only 7 bits are transferred to the subband filter stage. The subband filtering is done by 16 parallel polyphase bandpass FIR filters spanning the digital baseband of 0-75 MHz. The filter coefficients are represented using 9 bits and the output is 22 bit wide. A copy of each subband filter output is fed into a HT FIR filter (Q channel) and another is just phase shifted to match the phase shift due to HT filter (I channel). HT filter coefficients are 9 bit and the filter output is 35 bit wide. Therefore I channel is 22 bit wide and Q channel is 35 bit wide at the output of I/Q demodulation block. Only 7 most significant bits (MSB) are selected from both I and Q channels for further processing where cross-correlation $\left(I_{m} I_{n}\right.$, $\mathrm{Q}_{\mathrm{m}} \mathrm{Q}_{\mathrm{n}}, \mathrm{I}_{\mathrm{m}} \mathrm{Q}_{\mathrm{n}} \& \mathrm{Q}_{\mathrm{m}} \mathrm{I}_{\mathrm{n}}$ : subscript $\mathrm{m}, \mathrm{n}$ will be dropped hereafter) and moments $\left(1^{\text {st }}, 2^{\text {nd }}, 3^{\text {rd }} \& 4^{\text {th }}\right)$ are computed. This method of I/Q processing introduce a digital gain imbalance, Giq [14] which needs to be known for instrument calibration. Giq is the amount of scaling required for the $\mathrm{Q}$ channel to match I channel output. The theoretical Giq value
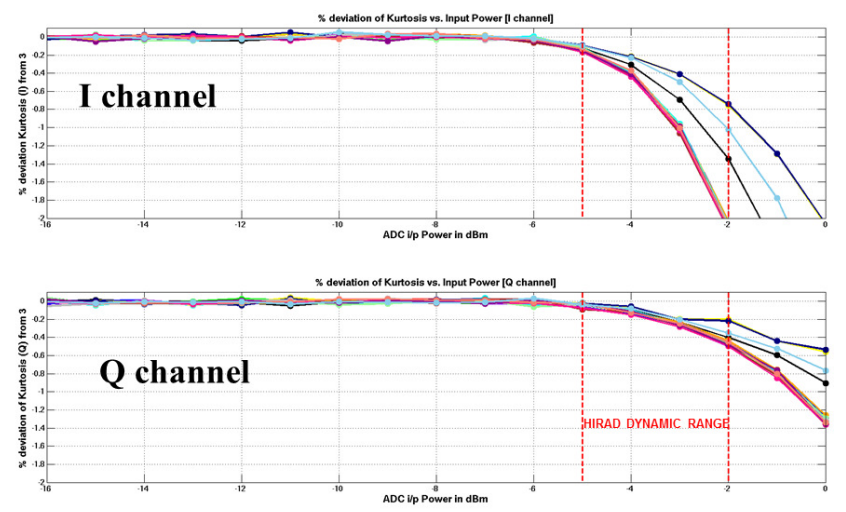

Figure 4: deviation (\%) of kurtosis vs. input power

of 1.2975 is obtained by considering the effect of scaling difference due to bit truncation and amplification of the $\mathrm{Q}$ channel signal due to Hilbert filter convolution. This value is very close to the value 1.296 obtained from instrument data.

\section{DBE SIMULATION}

A computer program is developed to simulate the HIRAD DBE signal processing including ADC quantization, bit truncation and finite bit arithmetic. A pair of discrete time partially correlated noise signals are generated in software whose power is controlled by changing the variance assuming a $50 \mathrm{ohm}$ system. The magnitude of the complex cross-correlation is set to 1 and the phase angle is varied to simulate different correlation for the real and imaginary part of the analytical signal. Thousand $\sim 9 \mathrm{~ms}$ integration periods are simulated for each power level between $-30 \mathrm{dBm}$ to +1 $\mathrm{dBm}$ in one $\mathrm{dBm}$ steps. It should be noted that the output power of HIRAD receivers were measured in the lab ( @ 30 $\operatorname{deg} C$ ) for all possible antenna switching states spanning the entire receiver dynamic range from cold load ( $\mathrm{min}$ ) warm load + noise diode (max). The dynamic range is between -5 to $-2 \mathrm{dBm}$ which included all 4 possible LO frequency. To verify the working of the simulator, the software generated noise signals were input (using an arbitrary waveform generator) to a spare HIRAD DBE running the same FPGA logic as the flight system. The FPGA recorded data agreed very well with the simulated data.

\subsection{Moments vs. Input Power}

The most important statistical moment of the signal is the second moment which is proportional to the power of the signal under measurement. Therefore the digital second moments from I and Q channels are a measurement of the system noise temperature from which the antenna temperature can be determined. The I and Q second moments from the same antenna are redundant measurements which can be combined to reduce measurement noise. Since there is a Giq scaling between the I and Q channels the scaling for the second moments should 

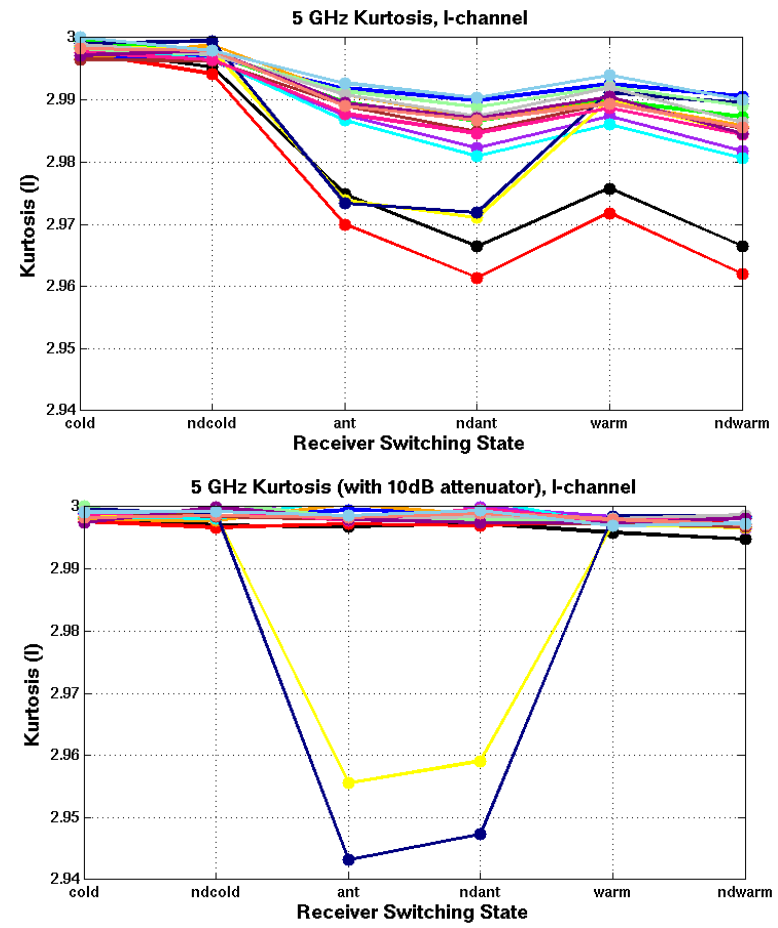

Figure 5: HIRAD $5 \mathrm{GHz}$ I-channel kurtosis for various receiver switching states in laboratory environment with interfering $5 \mathrm{GHz}$ WiFi signal (top). Same with a $10 \mathrm{~dB}$ attenuation of the IF signal before digitization.

be $\mathrm{Giq}^{2}$. The 1.296 value of the Giq was derived using second moments recorded from HIRAD flight data. In Fig. 2 , the $\%$ nonlinearity of second moment counts are plotted with respect to the input power for I and Q channel separately. Various colors are for 16 different subband channels. The HIRAD dynamic range is marked with two vertical dashed lines. Note that above $-3 \mathrm{dBm} \%$ deviation starts to deviate from 0 . Even at $-1 \mathrm{dBm}$ the non-linearity is less than $0.1 \%$. The Giq computed from simulated data matches perfectly with that observed real flight data except subbands \#1, \#8, \#9 and \#16 (bands near passband edges and center). Fig. 3 shows the $\%$ deviation of Giq from the value 1.296 for various input power. HIRAD dynamic range is in a "sweet spot" where Giq is essentially flat for most of the subbands.

The higher order moments are used along with the $2^{\text {nd }}$ moment to estimate kurtosis of the input signal. Natural thermal emission (i.e. Gaussian noise) has a kurtosis value equal to 3 . If the radiometric measurement is contaminated by manmade radio frequency interference (RFI) then the detected signal kurtosis can have any value. Therefore RFI which makes signal kurtosis other than 3 can be detected by HIRAD DBE. However, quantization affects kurtosis [] and its effect in HIRAD DBE is studied using the simulator. The simulated \% deviation of kurtosis from the ideal value 3 is plotted in Fig. 4 for various input power level. Note that the kurtosis has strong input power dependence in the HIRAD dynamic range. The $\mathrm{I}$ channel is affected more that the $\mathrm{Q}$
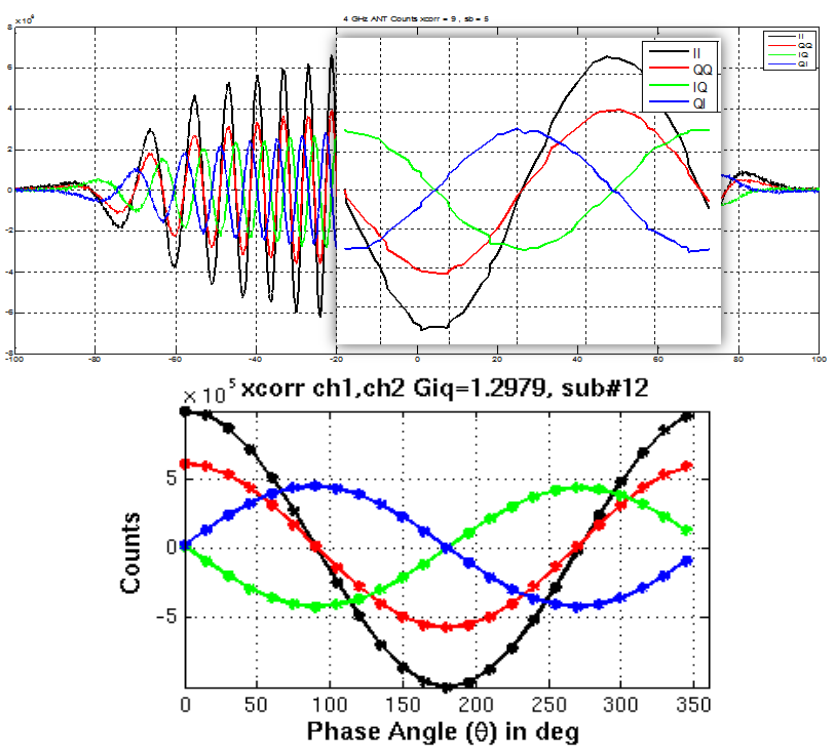

Figure 6: cross-correlation counts from chamber interference pattern measurement (top) and from the DBE simulator with varying phase angle of the input complex correlation coefficient (bottom).

channel because of the relative digital scaling between the two. The same phenomenon is also observed in HIRAD flight data where the $2^{\text {nd }}$ moment is found to be correlated with the kurtosis. Further, kurtosis showed a variation with the antenna switching states. HIRAD receiver switches between antenna, warm and cold load. For each of the switching state a noise diode (nd) signal is turned on and off creating six possible radiometer states. Top panel of Fig. 5 show the instrument kurtosis data collected in a lab environment for various switching states (30 sec average). Different colors are various subband channels. As expected from the DBE simulation, the kurtosis varies between receiver states. The bottom panel of Fig. 5 is plotted from data collected few minutes after with a $10 \mathrm{~dB}$ attenuator placed between the receiver and DBE. All the kurtosis values becomes very close to 3 for all receiver states. However, for subband \#8 and \#9 (center of the $5 \mathrm{GHz}$ RF passband) the antenna and antenna + nd signal kurtosis deviate significantly - which wasn't readily discernable in the data from original HIRAD configuration (Fig. 5, top panel). The lowering of the kurtosis value is found to be due to interference from the $5 \mathrm{GHz} \mathrm{WiFi}$ signal. Therefore, kurtosis sensitivity can be improved by lowering DBE input signal power as predicted by simulation results in Fig. 4.

\subsection{Cross-correlation vs. Input Power}

An unusual gain imbalance between II (QQ) and IQ (QI) counts was noticed during interference pattern measurement in the anechoic chamber against a radiated noise diode. Because of the Giq scaling between I and Q channel one would expect the maximum peak to peak swing of II to be 


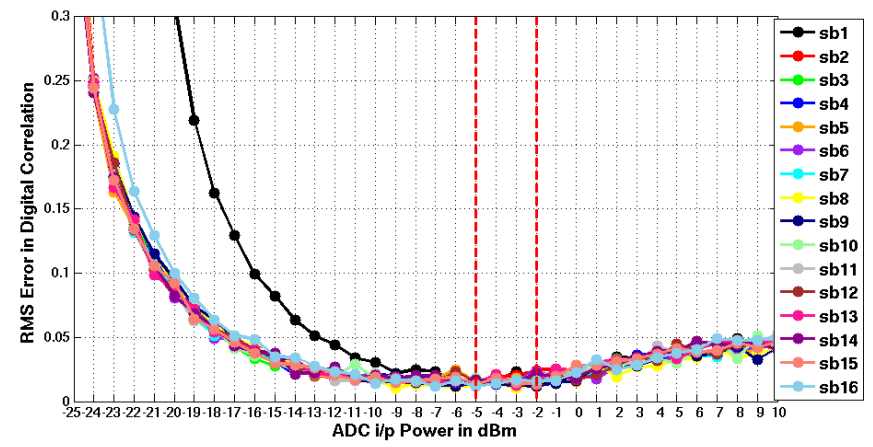

Figure 7: RMS error in digital correlation plotted against DBE input power

$\mathrm{Giq}^{2}$ times that of QQ and Giq times that of IQ (or QI). However, interference pattern counts from the chamber data showed that (top panel Fig. 6) IQ (QI) to be smaller than QQ. The relationship between II and QQ still held true. This behavior of the DBE wasn't expected but the simulator confirmed that this is a characteristic of the implemented DSP. In the Fig. 6 bottom panel the simulator output is plotted for various correlation input which show the exact same behavior observed during the chamber test. Next the normalized digital correlation is computed for various input analog correlation at different input power level. The RMS difference between analog and normalized digital correlation combining II, IQ, QI \& QQ is plotted in Fig. 7. In the HIRAD dynamic range (between the dashed vertical lines) The RMS error is below 0.025 for all subbands.

\section{CONCLUSIONS}

The performance of a radiometer digital back end (DBE) is analyzed here. The particular design studied here corresponds to the DBE of the airborne Hurricane Imaging Radiometer instrument. A computer simulator is developed to analyze effect of input power on various DBE output products. The $2^{\text {nd }}$ moment nonlinearity is found to be negligible in the HIRAD dynamic range. However, kurtosis statistics can be improved by lowering the DBE input power. Digital correlation error is found to be minimal in the HIRAD dynamic range. An unexpected scaling between $\mathrm{II}(\mathrm{QQ})$ and $\mathrm{IQ}(\mathrm{QI})$ observed during the interference pattern measurement is confirmed to be a DBE characteristics.

\section{REFERENCES}

[1] Thompson A. R., Moran J. M. and Swenson Jr. G. W., Interferometry and Synthesis in Radio Astronomy, $2^{\text {nd }}$ Edition, Chapter 8,Wiley 2004.

[2] Ruf, C.S., S. M. Gross and S. Misra, "RFI Detection and Mitigation for Microwave Radiometry with an Agile Digital Detector," IEEE Trans. Geosci. Remote Sens.,44(3), 694-706, 2006.
[3] Weinreb S. Digital radiometer. Proc. IEEE. 1961;49:1099.

[7] Cooper BF. Correlators with two-bit quantization. Aust. J. Phys. 1970;23:521-527.

[8] Van Vleck JH, Middleton D. The spectrum of clipped noise. Proc. IEEE. 1966;54:2-19.

[9] Price R. A useful theorem for nonlinear devices having Gaussian inputs. IRE Trans. Inform. Theory. 1958;4:69-72.

[10] Hagen J, Farley D. Digital-correlation techniques in radio science. Radio Sci. 1973;8:775-784.

[11] Piepmeier JR, Gasiewski AJ, Almodovar JE. Advances in microwave digital radiometry. Proc. IEEE Int. Geosci. Remot. Sens. Symp. 2000;7:2830-2833.

[13] Ruf CS. Digital correlators for synthetic aperture interferometric radiometry. IEEE Trans. Geosci. Remot. Sens. 1995;33:1222-1229.

[14] Ruf, C.; Roberts, J.B.; Biswas, S.; James, M.; Miller, T., "Calibration and image reconstruction for The Hurricane Imaging Radiometer (HIRAD)," Geoscience and Remote Sensing Symposium (IGARSS), 2012 IEEE International , vol., no., pp.4641,4643, 22-27 July 2012

[15] Ruf, C.; Amarin, R.; Bailey, M.C.; Boon Lim; Hood, R.; James, M.; Johnson, J.; Jones, L.; Rohwedder, V.; Stephens, K., "The hurricane imaging radiometer - an octave bandwidth synthetic thinned array radiometer," in Geoscience and Remote Sensing Symposium, 2007. IGARSS 2007. IEEE International, vol., no., pp.231-234, 23-28 July 2007

[16] Bailey, M.C., R. Amarin, J. Johnson, P. Nelson, M. James, D. Simmons, C. Ruf, L. Jones and X. Gong, "Multi-Frequency Synthetic Thinned Array Antenna for the Hurricane Imaging Radiometer," Trans. Antennas Propagat., 58(8), doi:10.1109/TAP.2010.2050453, 2010. 